\title{
REGULACIÓN DE LA INVESTIGACIÓN BIOMÉDICA EN CHILE
}

\author{
María Angélica Sotomayor Saavedra*
}

Resumen: Este artículo analiza el desarrollo normativo en Chile sobre investigación en seres humanos, a partir de 1990 y en el contexto de tratados de los derechos humanos. Se analiza la investigación científica desde el punto de vista del investigador y de las personas que sirven como sujetos, centrando la atención en la que utiliza productos farmacéuticos cuya comercialización no está autorizada en el país. Se examina también la evolución normativa relativa a la protección de los sujetos de investigación y las atribuciones para desarrollar tales ensayos en los establecimientos de salud. Se concluye con un análisis crítico de la regulación vigente y una mirada a los desafíos.

Palabras clave: investigación, regulación, derechos humanos, probando, investigador

\section{ADJUSTMENT OF BIOMEDICAL INVESTIGATION IN CHILE}

\begin{abstract}
This paper analyses the development of the normative on human beings' development in Chile since 1990 onwards considering the context of the human rights' treaties. Scientific investigation is analysed from the investigator's point of view and that of people serving as subjects. It focalizes its attentions on the one that employs pharmaceutical products that are not commercially authorized in the country. It also examines the normatives' evolution regarding the investigation's protections towards its subjects, and the attributions to develop these essays inside the health-care institutions. The work ends with a critical analysis of today's guidelines and with a glance at its challenges.
\end{abstract}

Key words: investigation, regulation, human rights, testing, researcher

\section{REGULAMENTAÇÃO DA PESQUISA BIOMÉDICA NO CHILE}

Resumo: Este artigo analisa o desenvolvimento normativo da pesquisa em seres humanos no Chile, a partir de 1990 e no contexto de tratados de direitos humanos. A pesquisa científica é analisada desde o ponto de vista do pesquisador e das pessoas que servem como sujeitos, centrando a atenção na pesquisa que utiliza produtos farmacêuticos cuja comercialização não sta autorizada no país. É examinada também a evolução normativa relativa à proteção dos sujeitos de pesquisa e as atribuições para desenvolver tias ensaios nos establecimentos de saúde. Conclui-se com uma análise crítica da regulação vigente e dos desafios.

Palavras chave: pesquisa, regulamentação, direitos humanos, probando, pesquisador

\footnotetext{
Abogada, Magíster en Derecho. Docente de la Facultad de Medicina, Universidad de Chile, Chile

Correspondencia: msotomay@med.uchile.cl
} 


\section{Introducción}

La historia de la humanidad muestra grandes transgresiones contra seres humanos bajo el pretexto de obtener conocimiento, y es precisamente la constatación de éstas la que dio origen en el siglo XX al surgimiento de la ética de la investigación en seres humanos.

Nuestra historia no da cuenta de hechos repudiables realizados por investigadores. La investigación científica se ha desarrollado tradicionalmente en el seno de las universidades a las que se han sumado en los últimos años centros e institutos de investigación. El organismo más importante de Chile en investigación científica es la Comisión Nacional de Investigación Científica y Tecnológica CONICYT, que no ha abordado el tema de la regulación. A través de concursos anuales de proyectos, se ocupa del financiamiento de la investigación científica en general, incluida la investigación biomédica en seres humanos.

Pese a que el Estado de Chile había suscrito y ratificado el Pacto Internacional de Derechos Civiles y Políticos de 1966, vigente a partir de 1976, con norma específica relativa a la experimentación con seres humanos, sólo a partir de 1990 el Ministerio de Salud dictó la primera norma sobre la materia como Directiva Permanente Interna.

\section{Investigación y derechos humanos}

La libertad de investigación, parte de la capacidad creadora del hombre, aparece consagrada como derecho en la Declaración Americana de los Derechos y Deberes del Hombre, aprobada en la Novena Conferencia Internacional Americana, Bogotá, 1948. Ha sido regulada en conjunto con las libertades de opinión, expresión y difusión del pensamiento por cualquier medio. También, la Declaración Universal de los Derechos Humanos de Naciones Unidas, de 1948, concibe la libertad de investigar como parte de la libertad de opinión y expresión, "derecho que incluye el de no ser molestado a causa de sus opiniones, el de investigar y recibir informaciones y opiniones, y el de difundirlas sin limitación de fronteras, por cualquier medio de expresión". Los primitivos desafíos en asegurar la libertad de investigación y de creación, reconocidos como derechos humanos en las Declaraciones señaladas, se han desplazado en los años recientes hacia la protección de las personas e, inclusive, a que el avance científico no afecte la biosfera y las generaciones futuras.
En el presente trabajo se analizará la relación regulatoria de los temas mayormente vinculados en la materia, a saber, la libertad de investigación y la protección de los sujetos de investigación, presupuesto necesario y a la vez dique de contención de la primera.

La investigación con seres humanos, desde la perspectiva de los derechos humanos, centra su atención en la protección de los sujetos de investigación y, eventualmente, de la población en general. En este sentido, tal forma de investigación se vincula prácticamente con todos los derechos y garantías constitucionales basados en el respeto fundamental a la dignidad humana. Está relacionada con el respeto a la vida y a la integridad física y psíquica de la persona; la protección de la vida del que está por nacer; la igual protección de la ley en el ejercicio de los derechos de las personas; el respeto y protección de la vida privada y pública y de la honra de las personas y de su familia; el derecho a la libertad personal y a la seguridad individual; el derecho a la protección de la salud; el derecho de propiedad sobre toda clase de bienes corporales e incorporales, y el derecho del autor sobre sus creaciones intelectuales.

Respecto de la libertad en investigación, Chile ha sido parte y ratificado diversos textos, declaraciones y tratados que la reconocen. Entre ellos, y en el contexto de la Declaración Universal de Derechos Humanos, el Pacto Internacional de Derechos Económicos Sociales y Culturales de 1966, por el cual los Estados parte se comprometen "a respetar la indispensable libertad para la investigación científica y para la actividad creadora”. En relación con la protección de las personas, en el mismo contexto está el ya citado Pacto Internacional de los Derechos Civiles y Políticos de Naciones Unidas, de 1966, el cual prescribe, en el artículo séptimo, que nadie será sometido a torturas ni a penas o tratos crueles inhumanos o degradantes, agregando que: "En particular, nadie será sometido, sin su libre consentimiento, a experimentos médicos o científicos". La plena vigencia de ambos tratados se alcanzó en 1976, al reunirse el número suficiente de ratificaciones de Estados que ellos establecían; sin embargo, dado el período histórico en que este hecho ocurrió, no hubo suficiente información y conciencia, al menos en lo que a investigación científica se refiere, de su necesario cumplimiento.

En materia de derechos humanos, la Constitución Política de Chile contiene una norma que permite incorporar a la misma el reconocimiento de derechos 
esenciales que emanan de la naturaleza humana, en la medida en que Chile suscriba y ratifique tratados internacionales relativos a tales derechos, los que, de esta manera, pasan a ser derecho vigente, constituyendo limitaciones al ejercicio de la soberanía.

En la década del 70 del siglo pasado, el Estado decide dar un impulso a la investigación científica generada en el país y, mediante la Ley No 16.746, crea la Comisión Nacional de Investigación Científica y Tecnológica CONICYT, con funciones de asesoría al Presidente de la República en el planeamiento, fomento y desarrollo de investigaciones en el campo de las ciencias, y de coordinación de actividades del sector público y privado relativas al desarrollo de la ciencia y la tecnología. Entre las funciones de CONICYT está el desarrollo de la investigación en el campo de las ciencias puras y aplicadas, administrando también el Fondo Nacional de Desarrollo Científico (FONDECYT), destinado a estimular y promover el desarrollo de la investigación científica y básica en el país.

Aun cuando originalmente CONICYT no consideró la revisión ética de los protocolos de investigación, a partir de 1991 fue incorporando gradualmente recomendaciones y exigencias en tal sentido, aplicables a los concursos que convoca. En los formularios de postulación de proyectos a financiamiento consideró la sugerencia de que el postulante sometiera previamente la investigación a la aprobación del Comité de Ética de su institución, reservándose FONDECYT, en caso contrario, el derecho de enviarlo en consulta a la instancia que estimare. Más adelante, en 1993 y 1996, exigió una declaración de la institución patrocinante que cautelara la dimensión ética de los proyectos; de no incluirla, la postulación era eliminada. En 1999 exigió un documento de consentimiento informado para los participantes; en 2001 un informe de aprobación del Comité de Ética de la institución patrocinante, exigencia que en 2005 hizo extensiva a cada una de las instituciones involucradas.

En 2005, FONDECYT creó la Comisión Asesora de Ética y Bióetica con las funciones de: elaborar documentos propios sobre aspectos bioéticos que apoyen a los investigadores en la elaboración de sus proyectos de investigación; disponer información bioética (incluyendo material online) para apoyar a los investigadores; elaborar flujogramas de seguimiento de las normas bioéticas para los proyectos FONDECYT que lo requieran, y asesorar a los Comités de FONDECYT que soliciten apoyo en aspectos bioéticos.

Esta Comisión se abocó también al análisis de proyectos de investigación con seres humanos en curso o en etapa de concurso, observando deficiencias con relación al consentimiento informado y discrepancias entre evaluaciones formuladas por diversos comités de ética. A partir de 2001, CONICYT y el Ministerio de Salud establecieron vínculos para priorizar la investigación en salud relacionada con las necesidades reales del país, establecidas en los Objetivos Sanitarios Nacionales, creándose el Fondo Nacional de Investigación y Desarrollo en Salud (FONIS).

La investigación biomédica se ha desarrollado en Chile en el seno de las universidades, clínicas e institutos de investigación sobre la base de la libertad investigadora, de la autorregulación, del resguardo ético institucional y de la autonomía investigadora de que están dotadas las primeras, de acuerdo con la Ley Orgánica Constitucional de Enseńanza (LOCE).

Como se señaló, si bien en Chile existe libertad para investigar, respecto de la investigación biomédica corresponde al Estado -también constitucionalmente- la protección de la salud de la población. Esta protección se hace efectiva: otorgando autorización excepcional para uso del producto, cuando se trata de investigación con fármacos sin autorización sanitaria; protegiendo a los pacientes de los establecimientos de salud, mediante autorización de la investigación -previo consentimiento de los sujetos de la misma- por el director del establecimiento; regulando los comités de ética, que deben conocer los proyectos de investigación y pronunciarse velando por el bienestar de los sujetos de la investigación, y dictando normas a las cuales debe sujetarse la investigación biomédica con seres humanos.

La forma en que se desarrollan las actividades señaladas será objeto de análisis en el presente artículo.

\section{Investigación con seres humanos utilizando productos farmacéuticos cuya comercialización no está autorizada en el país}

Corresponde al Instituto de Salud Pública (ISP), organismo del Estado, otorgar autorización para la comercialización y distribución de productos farmacéuticos. La autorización sanitaria se realiza sobre la base de la 
inclusión y asignación de un número en un registro que lleva dicha institución. El director del ISP tiene, a partir de 1989, la atribución de autorizar provisionalmente el uso, sin previo registro, de productos farmacéuticos para investigación científica o ensayos clínicos. Esta atribución está regulada en el Reglamento del Sistema Nacional de Control de Productos Farmacéuticos.

Desde la perspectiva de las personas sobre las cuales se realizará la investigación, los directores de los establecimientos de salud tienen la atribución de autorizar la realización de tales estudios, sin perjuicio del consentimiento que debe dar el paciente. Para ejercer esta atribución se han asesorado por comités y comisiones a los que, sobre la base de las regulaciones, les ha correspondido la función de velar por el bienestar de los sujetos de la investigación biomédica.

\section{Tipos de comités}

Originalmente, el conocimiento de problemas bioéticos en relación con la práctica asistencial en los establecimientos públicos de salud y de análisis de proyectos de investigación que involucraren seres humanos y se desarrollaran en tales establecimientos estuvo radicado en un solo tipo de comité o comisión que tenía funciones de asesoría a los directores de éstos. Desde 1999, el Ministerio de Salud separó las funciones asistenciales y de investigación de los comités, creando los de evaluación ético-científica ${ }^{1}$, y estableció la obligación de que los proyectos de investigación que usaren productos no registrados debían ser informados a los comités de evaluación ético-científica del hospital o del servicio de salud, en su caso, y aprobados por el ISP antes de ser llevados a la práctica.

\section{Regulación actualmente vigente}

Como se señaló, en la base del marco normativo está la Constitución Política del Estado, en diversos aspectos:

- En relación con el reconocimiento por el Estado de la libertad e igualdad en dignidad y derechos de los hombres, y del respeto a los derechos esenciales

1 Los comités de evaluación ético-científica fueron creados por el Decreto Supremo No 494 del 19-7-1999, Ministerio de Salud, que modificó los Arts. 91 y 107 del Reglamento Orgánico de los Servicios de Salud (D. S. No 42 de 1986), al que introdujo un Art. 92 bis. El mismo Decreto Supremo modificó el Art. 16 del Decreto Supremo No 1876 de 1995 del Ministerio de Salud, Reglamento del Sistema Nacional de Control de Productos Farmacéuticos. que emanan de la naturaleza humana, sean aquellos garantizados por la Constitución Política o por tratados vigentes ratificados por Chile.

- Al establecer la Constitución deberes del Estado de actuar al servicio de la persona humana, siendo su finalidad la promoción del bien común; dar protección a la población y promover la integración armónica de todos los sectores de la Nación, y asegurar el derecho de las personas a participar con igualdad de oportunidades en la vida nacional.

- Al establecer determinados derechos y deberes constitucionales, tales como el derecho a la vida y a la integridad física y psíquica de la persona; la protección de la vida del que está por nacer; el respeto y protección de la vida privada y pública de la persona; el derecho a la protección de la salud; el derecho de propiedad, en sus diversas especies, sobre toda clase de bienes corporales o incorporales, y el derecho del autor sobre sus creaciones intelectuales, aplicable este último especialmente respecto del investigador y del patrocinador, en lo cual también es aplicable el Pacto Internacional de Derechos Económicos Sociales y Culturales de la ONU, de 1966.

- En cuanto prohíbe someter a las personas a experimentos médicos o científicos sin su libre consentimiento, norma vigente en Chile desde 1976, consagrada en el Pacto Internacional de Derechos Civiles y Políticos de la ONU, de 1966.

En el plano legislativo, la fuente más importante es la denominada "Ley sobre la Investigación Científica en el Ser Humano, su Genoma y Prohíbe la Clonación Humana”, No 20.120. Se trata de un cuerpo jurídico que, en pocos artículos, regula una gran cantidad de materias que el legislador justifica en el artículo primero, señalando que la finalidad del cuerpo normativo es la protección del ser humano (y específicamente de la vida desde la concepción), de su integridad física y psíquica, y de su diversidad e identidad genética.

Las normas aplicables específicamente a la investigación científica y a la protección de los seres humanos en relación con la investigación son escasas. Se encuentran en los artículos 10 y 11 de la Ley y establecen la obligación de que toda investigación científica en seres humanos que implique algún tipo de intervención física o psíquica debe ser realizada por profesionales idóneos en la materia, justificar su objetivo y metodología y ajustarse a los términos de la ley. Se consagra la prohibición de 
desarrollarla cuando hubieren antecedentes que permitan suponer riesgo de destrucción, muerte o lesión corporal grave y duradera para el sujeto. Interpretando la norma, cabe entender, en sentido contrario, que puede desarrollarse la investigación si los antecedentes permiten suponer que no hay riesgo de destrucción, muerte o lesión corporal grave y duradera.

Como se puede observar, los requisitos de gravedad y durabilidad son copulativos, lo que demuestra que la redacción no es feliz, ya que, continuando con el análisis textual, podría ser aceptable una lesión grave pero que no fuere duradera, y así llegar a situaciones absurdas. Esta falencia legislativa se agrava por cuanto nada señala respecto de la relación riesgo/beneficio a que aluden las normas éticas internacionales sobre la materia. Sin embargo, estimamos que se alcanzará una equilibrada aplicación de la norma, por cuanto el conocimiento e informe de toda investigación científica biomédica se entrega a un comité ético-científico, cuyo pronunciamiento se exige como una obligación, al igual que la autorización expresa del director del establecimiento dentro del cual se efectuará la investigación (nada dice respecto de la investigación que se realice fuera del establecimiento). Se establece también como perentorio que toda investigación científica (y no necesariamente biomédica) en seres humanos debe contar con el consentimiento previo, libre e informado del sujeto o de quien deba suplir la voluntad de éste, de acuerdo con la ley.

El requisito de contar con consentimiento informado se entiende cumplido si la persona que debe prestarlo "conoce los aspectos esenciales de la investigación, especialmente su finalidad, beneficios, riesgos y procedimientos o tratamientos alternativos". Previamente, la persona debe recibir información adecuada, suficiente y comprensible respecto de la investigación, debiendo informársele también de su derecho "de no autorizar la investigación (se entiende que a su respecto) o de revocar su consentimiento en cualquier momento y por cualquier medio, sin que ello importe responsabilidad, sanción o pérdida de beneficio alguno". El acta de consentimiento debe firmarla quien lo otorga, el director responsable de la investigación y el director del centro o establecimiento donde ésta se desarrollará, quien actúa como ministro de fe. Las modificaciones de los términos de la investigación requieren la obtención de nuevo consentimiento, salvo que el comité que la haya aprobado estime que tales modificaciones son menores.
Con el fin de proteger la identidad genética, la Ley cautela la reserva de la información genética del ser humano, señalando la obligación de encriptar los datos del genoma que permiten la identificación de una persona para efectos de su almacenamiento y transmisión. Establece además la prohibición de solicitar, recibir, indagar, poseer y utilizar información sobre el genoma de una persona, salvo autorización expresa de ésta o casos autorizados en la Ley; la prohibición de realizar prácticas eugenésicas, con excepción de la consejería genética, y de discriminar arbitrariamente a las personas por razones basadas en el patrimonio genético; en el mismo sentido, la prohibición de utilizar los exámenes genéticos y análisis predictivos como formas de discriminación.

La ley establece otras prohibiciones no relacionadas necesariamente con la investigación, como la de cultivar tejidos y órganos, salvo con fines diagnósticos, terapéuticos o de investigación científica; la de destruir embriones humanos para obtener células troncales que den origen a tejidos y órganos, y la de realizar terapia génica, salvo que sea con fines de tratamiento de enfermedades o para impedir su aparición. Además, establece la imposibilidad de atribuirse o constituir propiedad sobre el genoma humano o sobre parte de él, y de patentar el conocimiento de la estructura de un gen y las secuencias totales o parciales de ADN. Permite, sin embargo, la patentabilidad de procesos tecnológicos derivados del conocimiento del genoma humano, incluidos los procesos diagnósticos o terapéuticos. Finalmente, la ley crea la Comisión Nacional de Bioética, encargada de asesorar a los distintos poderes del Estado en "asuntos éticos que se presenten como producto de los avances científicos y biotecnológicos en biomedicina, así como en las materias relacionadas con la investigación científica biomédica en seres humanos, recomendando la dictación, modificación y supresión de las normas que la regulen".

El incumplimiento a las normas de la Ley se sanciona a través de la creación de diversas figuras penales o delitos. Directamente relacionados con la investigación, los de falsificación del acta de consentimiento y el desarrollo de un proyecto de investigación científica biomédica en seres humanos o su genoma sin contar con las autorizaciones legales.

Dada la generalidad de sus términos, la Ley entrega al Ministerio de Salud el mandato de complementar o 
desarrollar sus contenidos mediante la dictación de un reglamento, lo que a esta fecha no ha ocurrido.

También de rango legal es la norma que, al estar contenida en el Código Sanitario, regula el registro sanitario de los productos farmacéuticos. Se consagra la atribución del director del ISP para autorizar el uso de productos farmacéuticos sin registro sanitario con fines de investigación científica y ensayos clínicos, que debe ejercerse necesariamente, previo informe del comité ético-científico del Servicio de Salud u hospital correspondiente, "conforme a las normas sobre ensayos clínicos realizados en seres humanos utilizando productos farmacéuticos, que apruebe el Ministerio de Salud mediante resolución”. La reglamentación vigente corresponde a la Norma General Técnica No 57 de 2001 del Ministerio de Salud, que revisaré más adelante.

Además de la Ley de investigación, existen normas jurídicas aplicables a aspectos específicos relacionados con la investigación científica biomédica, resultado de la nueva estructuración del sector público de salud, como consecuencia de la reforma concretada en la aprobación de las leyes respectivas a partir de 2004, que buscaba la reorientación de las políticas públicas de salud, hacia objetivos nacionales en ese ámbito. Uno de los pilares de la reforma era el respeto de los derechos de los pacientes, habiendo iniciado el trámite del paquete legislativo necesario para llevarla a cabo, precisamente con el proyecto de ley sobre dicha materia ${ }^{2}$.

La reforma cambió la conformación del sector público en salud, distinguiéndose y separándose, en el seno del Ministerio, las funciones relativas a la prestación de acciones que otorgan los servicios de salud -cuya supervisión quedó radicada en la Subsecretaría de Redes Asistenciales- de las funciones de protección de la salud y cumplimiento del Código Sanitario -cuya supervisión quedó radicada en la Subsecretaría de Salud Pública, de la que dependen las secretarías regionales ministeriales de salud que constituyen la Autoridad Sanitaria. La investigación científica en seres humanos, utilizando fármacos sin registro sanitario, involucra el

2 El Proyecto de Ley sobre Derechos y Deberes de los Pacientes en Salud, Boletín No 02727-11, fue retirado de tramitación por el Ejecutivo y reemplazado por el Proyecto de Ley que regula los derechos y deberes que tienen las personas en relación con acciones vinculadas con su atención en salud. Boletín N 4398 de 2006. Información disponible en http://www.bcn.cl, visitado el 10 de marzo de 2007. quehacer de ambas subsecretarías. La autorización del director del ISP para el uso de fármacos sin registro y la protección de los sujetos de investigación (que es tarea de los comités de evaluación ético-científica) corresponden al área de protección de la salud. En lo que atañe a las personas sobre las cuales recaerá la investigación y a los recursos y establecimientos que participarán, corresponde al quehacer de los servicios de salud encargados del otorgamiento de prestaciones de salud. A este respecto, el Reglamento Orgánico de los Servicios de Salud establece entre las funciones de los directores de establecimientos públicos de salud la de autorizar los protocolos de investigación científica biomédica en seres humanos que se desarrollen al interior del establecimiento. Se exige en este caso, en forma previa, el informe favorable del "comité éticocientífico correspondiente".

La acción de los comités de evaluación ético-científica debe ajustarse a la Norma General Técnica No 57 de 2001, que se encuentra vigente al no haber sido derogada por norma posterior y al no haberse dictado el reglamento que prevé la Ley de investigación. Ésta afectó a la Norma Técnica referida en relación con el acta de consentimiento que, de acuerdo con dicha ley, debe suscribirse ante el director del establecimiento en el cual se desarrollará la investigación. También, las alusiones que hace la Norma a los servicios de salud deben entenderse referidas en la actualidad a las secretarías regionales ministeriales de salud (SEREMI's), al haberse modificado la estructura del sector salud.

La labor de los comités de evaluación ético-científica es la que proporciona, tanto al director del ISP como a los directores de establecimientos, la fundamentación ética y técnica respecto de la protección de las personas en las que recaerá la investigación. Llama la atención que, no obstante la importancia que tiene la protección de las personas, la regulación esté contenida en una norma técnica, que tiene un rango jurídico inferior a un reglamento.

La Norma General Técnica No 57 de 2001 regula los estudios clínicos con agentes farmacológicos en seres humanos, en sus diversas fases:

- Los estudios de fase I consideran a los estudios de seguridad y farmacocinética, dirigidos a establecer el rango de dosis tolerada y se ejecutan primordialmente en individuos sanos y, excepcionalmente, en severamente enfermos (ausencia de tratamiento efectivo conocido). Los estudios preclínicos no se 
incluyen por desarrollarse en animales, pero son de gran importancia para fundamentar los estudios en humanos, especialmente los relativos a toxicidad y teratogenicidad, cuyos resultados deben adjuntarse.

- Los estudios fase II contemplan las fases IIA y IIB, esto es, estudios de eficacia y seguridad en personas seleccionadas y estudios piloto que representan la demostración más rigurosa de la eficacia y seguridad de un fármaco, respectivamente.

- Los estudios clínicos fase III consideran las fases IIIA y IIIB. Los primeros se realizan después de demostrada la eficacia terapéutica del fármaco; los segundos, durante el período de aprobación de éste para la población que lo requiere.

- Los estudios fase IV se realizan después que el medicamento o agente biológico ha sido aprobado para su venta.

De acuerdo con la fase en que se desarrolle el estudio clínico, los requisitos que debe analizar el Comité de Evaluación Ético-Científico varían, siendo permanentes en las fases que se realizan en seres humanos: consentimiento informado de la persona que participará en el estudio; compromiso del investigador responsable con las pautas éticas del anexo $\mathrm{N}^{\circ} 1$ de la Norma, basado en las pautas publicadas por $\mathrm{CIOMS}^{3}$; seguro o garantía suficiente para efectos adversos; informe de potenciales beneficios a los participantes; protocolo de investigación; estudio previo de toxicidad en animales, incluyendo carcinogenicidad y teratogenicidad; Investigational Drug Brochure, que incluya toda la información disponible sobre el producto a evaluar; informe del número de pacientes de ensayos previos y países, y cumplimiento de infraestructura y personal para realizar el estudio por parte del establecimiento.

La Norma Técnica regula también los requerimientos a los investigadores, las instituciones en que se realicen los ensayos y los patrocinadores, el rol del ISP, los comités de evaluación ético-científica y los compromisos respecto de capacitación de sus miembros.

\section{Comités de evaluación ético-científica}

Corresponde al comité la evaluación de los aspectos

3 Council for International Organizations of Medical Sciences (CIOMS). International Ethical Guidelines for Biomedical Research Involving Human Subjects. Geneva: CIOMS; 2002. Hay versión en español: CIOMS. Pautas Éticas Internacionales para la Investigación Biomédica en Seres Humanos. Santiago de Chile: Programa Regional de Bioética OPS/OMS: 2003. éticos y científico-técnicos del proyecto, y auditar la ejecución del estudio clínico, conforme al protocolo aprobado.

De acuerdo con la Norma General Técnica No 57 de 2001, "toda institución donde se realice un protocolo de investigación que incluya a seres humanos que recibirán un fármaco en fase de investigación debe solicitar a un Comité de Evaluación Ético-Científico local que revise los aspectos científico-técnicos y éticos del protocolo de investigación, de los investigadores, del sitio donde se realice la investigación y de la institución patrocinadora". Como vimos, el requisito de contar con el pronunciamiento previo del Comité se exige para que el director del ISP autorice la internación y uso de un fármaco sin registro sanitario previo $y$, también, para que el director del establecimiento público de salud autorice la realización de un estudio con pacientes del establecimiento. En el punto 2.4 de la Norma se atribuye al investigador la obligación de presentar el proyecto al Comité.

A falta del Comité de Evaluación Ético-Científico en el servicio de salud, debe designarse un Comité de Evaluación Ético-Científico de Referencia. La Norma 57 alude a los comités de los servicios de salud pero, como ya señalamos, la nueva estructura del sector salud radica estas materias en las SEREMI's que constituyen la Autoridad Sanitaria.

Tratándose de estudios que sobrepasen el ámbito de tres o más servicios de salud, o de estudios que puedan tener importancia estratégica para las políticas generales de salud del país, el Ministerio de Salud debe designar comités de evaluación ético-científicos ad hoc. Se encomienda también a dicho Ministerio la mantención de un registro de comités en funciones, con un sistema de acreditación, y de protocolos en ejecución, debiendo realizar revisiones periódicas de su desarrollo.

\section{Integración del Comité}

Lo integra un mínimo de ocho miembros, debiendo considerarse a representantes de ambos sexos, médicos y otros profesionales de la salud y juristas, uno con conocimientos de metodología y un representante de la comunidad. Los integrantes pueden provenir de la institución o de organismos externos, como facultades de medicina, sociedades científicas, entre otros. Son nombrados por "la autoridad del nivel correspondiente", cada cuatro ańos, en razón de la experiencia científica 
y ética, pudiendo ser redesignados sólo por una vez. El Comité debe designar un presidente y un secretario, y puede convocar a expertos en caso necesario. Deben actuar libres de conflictos de interés y comprometerse a cumplir cabalmente su labor.

\section{Procedimiento}

El presidente del Comité debe recibir los documentos del investigador, en castellano y en idioma original, si éste no fuere el castellano. Tratándose de la información al paciente y del formulario de consentimiento, siempre deben estar en castellano. Debe adjuntar pronunciamientos éticos respecto del proyecto o del producto. En el plazo de diez días seguidos, el presidente debe acusar recibo, si la documentación está conforme, o devolver los antecedentes al investigador si faltaren antecedentes, señalándolo así. El Comité debe pronunciarse dentro de los sesenta días corridos siguientes, aprobando o rechazando el proyecto, fundándose en razones científico-técnicas o éticas.

La aprobación o el rechazo procede por consenso, aceptándose sólo un voto de minoría. El acta debe suscribirse por los miembros del Comité. El estudio puede ser rechazado o bien objetado, lo que se informa al investigador, el que tiene quince días corridos para salvar las objeciones o presentar nuevos elementos. Presentados éstos, el Comité tiene el plazo de treinta días corridos para la decisión final, que se comunica al Ministerio de Salud y al investigador. De lo anterior se concluye que el pronunciamiento del Comité es vinculante para la autoridad.

\section{Seguimiento de la investigación}

El Comité debe conocer las enmiendas al protocolo, la evaluación de efectos adversos, resultados finales y cualquiera otra materia de interés, y debe evaluar, al menos una vez al año, la marcha de la investigación. Si estimare necesario suspender el desarrollo de ésta, debe citar al investigador para evaluar la situación y recomendar a la autoridad del establecimiento modificar la autorización o suspenderla. Dicha autoridad tiene quince días corridos para dar a conocer su decisión. El Comité también debe informar al Ministerio de Salud, el que debe pronunciarse en el mismo plazo.

El Comité debe resguardar la confidencialidad de la información y mantener un archivo de documentos relevantes por el término de tres años.
Se encarga a las autoridades respaldar la labor de los comités y otorgar las facilidades y estímulos necesarios para que cumplan sus funciones "de manera responsable y comprometida”.

\section{Análisis crítico de la regulación vigente}

Las normas vigentes en nuestro país en materia de investigación biomédica en seres humanos presentan deficiencias, algunas de las cuales se refieren a que:

- La regulación se encuentra dispersa en distintas normas, de diversa jerarquía, estando contenida la más específica en una norma jurídica de rango inferior, como es el caso de una Norma Técnica.

- La ley de Investigación No 20.120 de 2006 es imprecisa en cuanto a qué tipo de investigación en seres humanos regula. Sólo en los artículos 10 y 11 considera las normas específicamente aplicables a la investigación biomédica con seres humanos.

- Las normas apuntan a autoridades y autorizaciones, esto es, a procedimientos para hacer efectivos los estudios de investigación, pero nada dicen respecto de la protección de las personas. Sólo la Norma Técnica No 57 hace aplicables las declaraciones internacionales sobre ética de la investigación para hacer efectiva la protección de los sujetos de la investigación biomédica.

- La regulación y en especial la Norma Técnica No 57, al complementar la atribución del director del ISP para autorizar el uso de productos farmacéuticos sin registro sanitario previo, no incluye otras investigaciones en seres humanos, por ejemplo con fármacos ya autorizados pero para un uso distinto del registrado, o para investigar una vía distinta de administración de la droga, o investigaciones con elementos de uso médico, entre otras.

- No se observa coordinación entre la regulación relativa a los comités y la atribución del director del ISP.

- La nueva estructura del sector salud no consideró una organización dedicada a la coordinación de la investigación con seres humanos.

- La Norma no hace referencia explícita a las investigaciones que se llevan a cabo con pacientes de establecimientos privados ni a la que se realiza en las consultas profesionales.

- La regulación se refiere, en general, a estudios provenientes de la industria farmacéutica internacional, materia que sin duda es importante pero no 
aplicable necesariamente a los estudios que tienen origen nacional, como es el caso, por ejemplo, de los originados en las universidades y financiados por éstas, por otros entes o por fondos nacionales para investigación, salvo que estos estudios requieran la internación al país de productos farmacéuticos no registrados.

- La regulación no resguarda debidamente la información sensible que surja de los sujetos de investigación, materia que debiera estar especialmente normada y no sujeta a la convención.

- Asuntos tan importantes como la protección económica de los sujetos de investigación, a través de la exigencia de seguros que resguarden los riesgos de efectos adversos, incapacidad o muerte del sujeto, están considerados no en la parte principal de la Norma sino en el anexo № 1 .

- Los estudios provenientes del extranjero contienen exigencias aplicables en esos países conformes con su sistema jurídico. La información al paciente y el formulario de consentimiento informado tienen la forma de un convenio de varias páginas con mucha información, a veces ininteligible para el sujeto, lo que hace que su comprensión deba ser mediada por el investigador.

- Entendiendo que la regulación nacional no requiere repetir los consensos logrados respecto de la protección de los sujetos de investigación en las normas éticas internacionales, algunas situaciones requieren ser adaptadas a la cultura nacional. Eso ocurre, por ejemplo, con el asentimiento del incapaz y el consentimiento de su representante: los estudios extranjeros aclaran que debe otorgarlo el representante legal o el designado por el juez. En nuestro sistema sanitario tal autorización suele darla la persona que tiene a cargo al incapaz. Otra duda surge cuando se trata de madres menores de edad respecto de autorizar a sus hijos para la investigación biomédica.

- Lo mismo ocurre con la regulación y la vulnerabilidad a la que puede estar afecta una parte de la población, por ejemplo, en razón de no disponer de recursos sanitarios de última tecnología y ver en los estudios multicéntricos la posibilidad de acceder a ellos. Esto que puede ser una amenaza, si las personas están bien protegidas puede representar una oportunidad. Sin embargo, nuestra experiencia en la revisión de estudios multicéntricos provenientes del extranjero nos muestra que, cada día más, los sujetos seleccionados para estudios provienen del segmento de la población con mayor disponibilidad de recursos y educación. Ello dados los medios que se les pone a su alcance, por ejemplo, teléfonos celulares y otras tecnologías, para facilitar el seguimiento de los estudios por parte de los investigadores o de las empresas coordinadoras de la investigación, también denominadas Organización de Investigación por Contrato (CRO, en su sigla en inglés). En concreto, tal situación infringiría el principio de justicia, al tratarse de una situación de discriminación.

- Existen otros vacíos regulatorios, ni siquiera considerados en las declaraciones internacionales sobre la materia, relativos, por ejemplo, a la globalización de la investigación biomédica, ya no entre patrocinadores (esto es, la industria) sino a través de instituciones concertadas en el extranjero para desarrollar investigación biomédica con seres humanos - por ejemplo, industria-universidades, o bien universidades con grupos de investigadores-, los que ofician en nuestro país como patrocinadores e investigadores al mismo tiempo, con los consiguientes conflictos de interés. Otra situación compleja es la asociación entre pacientes y corporaciones, o la existencia de fundaciones que reúnen pacientes y negocian por ellos con los patrocinadores, perdiéndose la necesaria independencia del sujeto de investigación.

- Otro vacío se refiere a las organizaciones que coordinan la investigación (CRO), empresas que intermedian con los patrocinadores extranjeros, no siempre domiciliados en Chile, y que contratan localmente a los investigadores. El problema es que la relación patrocinador-investigador está mediada por un tercero y estas organizaciones podrían no contar con patrimonio suficiente para responder por eventuales dańos a los sujetos de la investigación.

- No están regulados los estudios con fines promocionales o los que se realizan sobre la base de una prestación de servicios.

- En una ética de la investigación basada en los derechos humanos, como corresponde de acuerdo con la Constitución Política, el Estado debiera resolver en su regulación nacional materias tan importantes como el uso del placebo, uso compasivo, eventuales beneficios para los sujetos de la investigación, distinción de la investigación con 
beneficio para el sujeto respecto de aquella que no lo tiene, eventuales acuerdos que podría obtener el país como Estado respecto de determinadas investigaciones, la obligación de publicar los resultados de los estudios, definición de investigaciones que son relevantes para la población, resguardo especial para los estudios de farmacogenética o farmacogenómica que, en la actualidad, se presentan como apéndices de otros estudios, entre otras materias.

\section{Compromisos futuros como Estado}

El escenario jurídico y ético en nuestro país demuestra que, si bien existe la base constitucional y los pactos internacionales de derechos civiles y políticos, y de derechos económicos, sociales y culturales -con norma expresa aplicable a la investigación, el primero-, recién con la dictación de la Ley de investigación se ha iniciado el desarrollo legislativo sobre la materia, al cual debe integrarse el reglamento previsto en la ley citada, cuya redacción se encomienda al Ministerio de Salud. Sin embargo, dicha autoridad no puede ignorar que existen otros actores involucrados en la materia, como ocurre con CONICYT, las universidades y la sociedad civil en general.

Desde otro punto de vista, existen compromisos del Estado en el ámbito del Derecho Internacional Público relativo a los derechos humanos, contraídos en la Declaración Universal sobre el Genoma Humano y en la Declaración Universal sobre Bioética y Derechos
Humanos, ambos de UNESCO. Algunos de estos compromisos son:

- La promoción de comités de ética independientes, pluridisciplinarios y pluralistas en relación con las investigaciones relativas a los seres humanos y sobre el genoma humano. A ello debiera agregarse que cuenten con los medios y recursos para asegurar su adecuado funcionamiento e independencia.

- El aprovechamiento compartido de los beneficios de la investigación científica y sus aplicaciones, especialmente para las personas que hayan tomado parte en la investigación.

- La protección a las generaciones futuras, al medio ambiente, la biosfera y a la biodiversidad.

- El fomento de investigaciones dirigidas a identificar, prevenir y tratar enfermedades relacionadas con la genética y enfermedades raras o endémicas que afectan a parte considerable de la población.

- El sometimiento de las investigaciones transnacionales a un nivel adecuado de examen ético del país anfitrión, la necesidad de responder a los requerimientos de salud de éste y de establecer condiciones de colaboración y acuerdo sobre los beneficios de la investigación, con participación equitativa de las partes.

También es parte de los compromisos que tales propósitos debieran lograrse sobre la base del diálogo multidisciplinario, pluralista e informado de la sociedad en su conjunto, como se espera ocurra en una sociedad democrática y participativa.

\section{Bibliografía}

Alvarez J, Lolas F, Outomuro D. Historia de la ética en investigación con seres humanos, En: Lolas F, Quezada A, Rodríguez E. Investigación en Salud. Dimensión Ética. Santiago de Chile: CIEB, Universidad de Chile; 2006: 39-46.

Código Sanitario. Art. 102. Santiago de Chile: Editorial Jurídica Cono Sur; 2001: 102.

Consejo de Organizaciones Internacionales de las Ciencias Médicas (CIOMS). Pautas Éticas Internacionales para la Investigación Biomédica en Seres Humanos. Santiago de Chile: Programa Regional de Bioética OPS-OMS; 2003.

Constitución Política de la República de Chile. Arts 5, 19, Diario Oficial de 22-09-2005.

Ley $N^{o}$ 16746. Diario Oficial de 14-02-1968.

Ley No 20.120 "Sobre la Investigación Cientifica en el Ser Humano, su Genoma y Prohibe la Clonación Humana". Diario Oficial de 22-9-2006.

Ley Orgánica Constitucional de Enseñanza No 18.962. Texto refundido fijado por DFL. № 1 de 24-12-2005, Diario Oficial del 29-2-2006.

Mensaje Boletín No 2980-11. Proyecto de ley que se concreta como Ley No 19937. (Sitio en Internet) disponible en http://www. bcn.cl Acceso el 10 de marzo de 2007.

Ministerio de Salud de Chile. Directiva Permanente Interna No 10. Santiago de Chile: Biblioteca Asesoría Jurídica Minsal: 1990. 
Ministerio de Salud. Decreto Supremo No 494 de 19-7-1999. MINSAL, Diario Oficial de 14-11-2000.

Ministerio de Salud. Norma General Técnica No 57. Aprobada mediante Resolución Exenta No 952 de 4-06-2001.

Ministerio de Salud. Reglamento del Sistema Nacional de Control de Productos Farmacéuticos. Decreto Supremo No 1876 de 1995. MINSAL, Diario Oficial de 29-9-1996.

Ministerio de Salud. Reglamento Orgánico de los Servicios de Salud. Aprobado por Decreto Supremo No 140. MINSAL, Diario Oficial de 21-4-2005.

Naciones Unidas. Declaración Americana de los Derechos y Deberes del Hombre, 1948. En: Pacheco M. Los Derechos Humanos. Documentos Básicos. Santiago de Chile: Editorial Jurídica de Chile; 1987: 53-58.

Naciones Unidas. Declaración Universal de los Derechos Humanos, 1948. En: Pacheco M. Los Derechos Humanos. Documentos Básicos. Santiago de Chile: Editorial Jurídica de Chile; 1987: 9-65.

Naciones Unidas. Pacto Internacional de Derechos Civiles y Políticos, 1966. Art. 7. En: Pacheco M. Los Derechos Humanos. Documentos Básicos. Santiago de Chile: Editorial Jurídica de Chile; 1987: 158-179.

Naciones Unidas. Pacto Internacional de Derechos Económicos Sociales y Culturales, 1966. En: Pacheco M. Los Derechos Humanos. Documentos Básicos. Santiago de Chile: Editorial Jurídica de Chile; 1987: 147-157.

UNESCO. Declaración Universal sobre Bioética y Derechos Humanos, octubre de 2005 (Sitio en Internet) Disponible en http://www.unesco.org/shs/ethics Acceso el 15 de marzo de 2007.

UNESCO. Declaración Universal sobre el Genoma Humano y los Derechos Humanos, noviembre de 1997. (Sitio en Internet) Disponible en http://www.unesco.org/shs/ethics Acceso el 15 de marzo de 2007.

Velasco P. Aspectos Bioéticos de la Investigación Cientifica de Proyectos. Santiago de Chile: CONICYT-FONDECYT; 129-130. (Sitio en Internet) Disponible en http://www.conicyt.cl Acceso el 28 de febrero de 2007.

Recibido: 4 de septiembre de 2007

Aceptado: 4 de octubre de 2007 